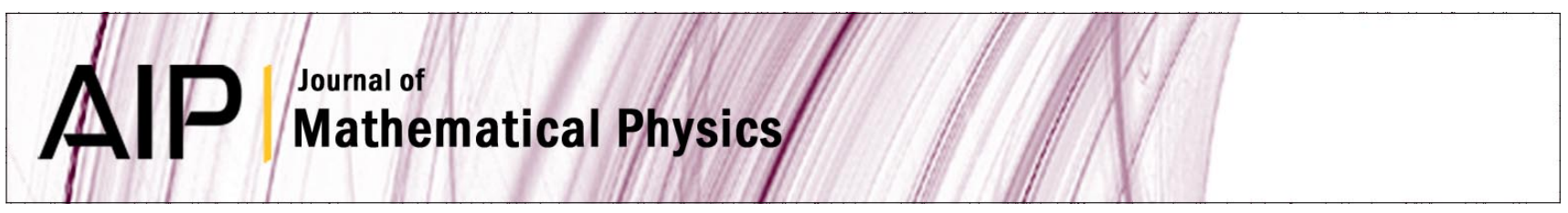

\title{
Quantal effects and MaxEnt
}

F. Holik and A. Plastino

Citation: J. Math. Phys. 53, 073301 (2012); doi: 10.1063/1.4731769

View online: http://dx.doi.org/10.1063/1.4731769

View Table of Contents: http://jmp.aip.org/resource/1/JMAPAQ/v53/i7

Published by the American Institute of Physics.

\section{Related Articles}

Existence of the thermodynamic limit for disordered quantum Coulomb systems J. Math. Phys. 53, 095209 (2012)

Superfluid ultra-dense deuterium $\mathrm{D}(-1)$ on polymer surfaces: Structure and density changes at a polymer-metal boundary

J. Appl. Phys. 111, 123502 (2012)

Optimizing hierarchical equations of motion for quantum dissipation and quantifying quantum bath effects on quantum transfer mechanisms

J. Chem. Phys. 136, 224103 (2012)

Continuum limit frozen Gaussian approximation for the reduced thermal density matrix of dissipative systems

J. Chem. Phys. 136, 094101 (2012)

Numerical Feynman integrals with physically inspired interpolation: Faster convergence and significant reduction of computational cost

AIP Advances 2, 012121 (2012)

\section{Additional information on J. Math. Phys.}

Journal Homepage: http://jmp.aip.org/

Journal Information: http://jmp.aip.org/about/about_the_journal

Top downloads: http://jmp.aip.org/features/most_downloaded

Information for Authors: http://jmp.aip.org/authors

\section{ADVERTISEMENT}

The most comprehensive support for physics in any mathematical software package World-leading tools for performing calculations in theoretical physics

Your work in Maple matches how you would write the problems and solutions by hand

- State-of-the-art environment for algebraic computations in physics

The only system with the ability to handle a wide range of physics computations as well as pencil-and-paper style input and textbook-quality display of results

- Access to Maple's full mathematical power, programming language, visualization routines, and document creation tools 


\title{
Quantal effects and MaxEnt
}

\author{
F. Holik ${ }^{1, a)}$ and A. Plastino ${ }^{2}$ \\ ${ }^{1}$ Departamento de Matemática, Ciclo Básico Común, Universidad de Buenos Aires, \\ Pabellón III, Ciudad Universitaria, Buenos Aires, Argentina \\ ${ }^{2}$ National University La Plata and CONICET IFLP-CCT, C.C. 727-1900 La Plata, \\ Argentina; Universitat de les Illes Balears and IFISC-CSIC, 07122 Palma de Mallorca, \\ Spain; and Instituto Carlos I de Fisica Teorica y Computacional and Departamento de Fisica \\ Atomica, Molecular y Nuclear, Universidad de Granada, Granada, Spain
}

(Received 25 August 2011; accepted 13 June 2012; published online 11 July 2012)

Convex operational models (COMs) are considered as great extrapolations to larger settings of any statistical theory. In this article, we generalize the maximum entropy principle (MaxEnt) of Jaynes' to any COM. After expressing MaxEnt in a geometrical and lattice theoretical setting, we are able to cast it for any COM. This scope-amplification opens the door to a new systematization of the principle and sheds light into its geometrical structure. (C) 2012 American Institute of Physics. [http://dx.doi.org/10.1063/1.4731769]

\section{INTRODUCTION}

The notion of using a small set of relevant expectation values so as to describe the main properties of physical systems may be considered the leit-motiv of statistical mechanics. ${ }^{1}$ Developments based upon Jaynes' maximum entropy principle (MaxEnt) constitute a pillar of our present understanding of the discipline. ${ }^{2,3}$ This type of ideas has also been invoked for obtaining the probability distribution associated with pure quantum states via MaxEnt (see, for instance, Ref. 4 and references therein). Indeed, MaxEnt constitutes a very important physical viewpoint, on the one hand, and powerful technique on the other one. This is true not only for physics, chemistry, astronomy, and engineering but for a host of other disciplines as well..$^{5}$ Thus, MaxEnt extensions that amplify its range of applicability should be regarded as significant for all branches of science.

In this article, we wish to introduce the powerful convex operational models (COM) approach $^{6-8}$ into the MaxEnt domain. COM is, in turn, intimately linked to convex sets of probability measures. ${ }^{6-11}$ Among the several extant approaches to the study of convex sets of probability measures, the above cited references deal with the COM-technique, in which physical states (understood as probability measures) and their convex structure play a key role, while other related quantities emerge in rather natural fashion. There exist generalizations of quantum mechanics, including nonlinear versions, that are axiomatized using the convex structure of the set of states (see Refs. 12-14). Using convex sets, one treats in geometrical fashion the statistical theory of systems and can also include quantum and classical mechanics (indeed several other theories as well). Elementary (sharp) tests in quantum mechanics are represented by projection operators that form the well-known von Newmann's lattice, an orthomodular one. ${ }^{15}$ Via the Born's rule any projection operator defines probabilities. These are linked to measures over the von Newmann's lattice: using Gleason's theorem, it is possible to link in a bijective way density matrixes and probability measures on the lattice of projections. But probability measures may also arise in quantum mechanics by means of positive operator valued measures (POVMs), which are also known as generalized measures. POVMs are also generalized easily to any COM. We will review these subjects in Sec. III.

We will in this effort cast Jaynes's MaxEnt approach in lattice theoretical form, which allows one to extend MaxEnt to any arbitrary convex operational model, which entails a considerable

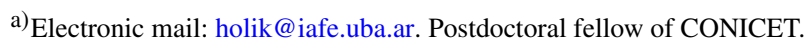


enlargement of its scope. Consequently, this extension provides a reformulation of the maximum entropy principle within the framework of the COM approach, which yields a novel perspective on the subject. One of the advantages of this extension/reformulation is that it is done in such a way that it allows to include conditions on POVMs, that is, to extend MaxEnt to generalized measurements, an important quantum mechanics' issue. Our generalization is performed in Secs. IV and V and should undoubtedly be of interest to thousands of MaxEnt practitioners. Since COMs constitute important extrapolations to larger settings of arbitrary statistical theories, casting MaxEnt for any COM, after expressing Jaynes' principle in a geometrical and lattice theoretical scenario, will hopefully open the door to a new systematization of the principle while shedding light into its geometrical structure.

\section{MAXIMUM ENTROPY PRINCIPLE}

Statistical mechanics and thereby thermodynamics can be formulated on the basis of information theory if the density distribution $\rho(x)$ is obtained by recourse to MaxEnt. ${ }^{2,3}$ Jaynes' stance asserts that assuming that your prior knowledge about the system is given by the values of $n$ expectation values of physical quantities $R_{j}$, i.e., $\left\langle R_{1}\right\rangle, \ldots,\left\langle R_{n}\right\rangle$, then the most adequate choice for the probability distribution $\rho(x)$ of our system is uniquely fixed by extremizing Shannon's logarithmic entropy $S$ subject to the $n$ constraint

$$
\left\langle R_{i}\right\rangle=r_{i} \text {; for all } i .
$$

This brings into the game $n$ Lagrange multipliers $\lambda_{i}$. In the process of employing the MaxEnt procedure, one discovers that the information quantifier $S$ can be identified with the equilibrium entropy of thermodynamics if our prior knowledge $\left\langle R_{1}\right\rangle, \ldots,\left\langle R_{n}\right\rangle$ refers to extensive quantities. ${ }^{2}$ $S$ (maximal), once determined, yields complete thermodynamical information with respect to the system of interest. ${ }^{2}$ The MaxEnt probability distribution function, associated with BoltzmannGibbs-Shannon's logarithmic entropy $S$, is given by $^{2,3}$

$$
\rho_{\max }=\exp \left[\left(-\lambda_{0} \mathbf{1}-\lambda_{1} R_{1}-\cdots-\lambda_{n} R_{n}\right)\right],
$$

where the $\lambda$ 's are Lagrange multipliers guaranteeing that

$$
r_{i}=-\frac{\partial}{\partial \lambda_{i}} \ln Z
$$

while the partition function reads

$$
Z\left(\lambda_{1} \cdots \lambda_{n}\right)=\exp ^{-\lambda_{1} R_{1}-\cdots-\lambda_{n} R_{n}},
$$

and the normalization condition

$$
\lambda_{0}=\ln Z .
$$

Such simple-looking algorithm constitutes one of the most powerful ones in physics' arsenal. In a quantum setting, of course, the $R$ 's are operators on a Hilbert space $\mathcal{H}$, while $\rho$ is a density matrix (operator).

\section{COM-PRELIMINARIES}

We recapitulate here essential aspects of convex operational models. $\mathcal{P}(\mathcal{H})$ will denote the set of all closed subspaces of $\mathcal{H}$, which are in a one-to-one correspondence with the projection operators. Because of the one-to-one link, one usually employs the notions of "closed subspace" and "projector" in interchangeable fashion. The bounded operators on $\mathcal{H}$ will be denoted by $\mathcal{B}(\mathcal{H})$. We begin the present task with reference to classical probabilities. Given a set $\Omega$, let us consider a $\sigma$-algebra $\Sigma \subseteq \mathcal{P}(\Omega)$. Then, a probability measure will be given by a function

$$
\mu: \Sigma \rightarrow[0,1],
$$


which satisfies the well-known axioms of Kolmogorov. ${ }^{16}$ We remind that in the traditional formulation of quantum mechanics, states may be defined as functions of the form ${ }^{17,18}$

$$
s: \mathcal{P}(\mathcal{H}) \rightarrow[0 ; 1]
$$

such that

1. $s(\mathbf{0})=0(\mathbf{0}$ is the null subspace);

2. $S\left(P^{\perp}\right)=1-s(P)$;

3. For any pairwise orthogonal denumerable family of projections $P_{j}$ one has $s\left(\sum_{j} P_{j}\right)=\sum_{j} s\left(P_{j}\right)$.

For a reference on the distinction between classical and quantum probabilities the reader is referred to Chap 2 of Ref. 11. Relatively, recent discussions about the nature of probabilities in quantum mechanics clearly demonstrate that relevant features can be derived in an operational setting which is closer in spirit to standard probability theory and Boolean logic. ${ }^{19,20}$ This is done by postulating that to any process one is to (i) associate a pair of real numbers (a quite natural assumption inspired by the principle of complementarity) and then (ii) use a method first developed by Cox (Refs. 21 and 22) -in which the probability calculus can be regarded a generalization of the Boolean logic of propositions- to derive Feyman's rules. ${ }^{19}$ In this article, the above mentioned distinction plays no special role, since we are only concerned with the geometrical and formal structure of the space of quantum states given by (7). In order to review its main geometrical features, let us remind that Gleason's theorem ${ }^{23,24}$ asserts that if $\operatorname{dim}(\mathcal{H}) \geq 3$, then the set of all measures of the form (7) can be put into a one-to-one correspondence with the set $\mathcal{C}$ of by all positive, hermitian, and trace-class (normalized to unity) operators in $\mathcal{B}(\mathcal{H})$. If $P \in \mathcal{P}(\mathcal{H})$, the correspondence between $\rho \in \mathcal{C}$ and its induced probability measure is given by

$$
s_{\rho}(P)=\operatorname{tr}(\rho P),
$$

where $\operatorname{tr}(\cdots)$ stands for the trace operator in $\mathcal{B}(\mathcal{H})$, i.e., the sum of all the eigenvalues. Equation (8) is essentially Born's rule. Any $\rho \in \mathcal{C}$ may be written as

$$
\rho=\sum_{i} p_{i} P_{\psi_{i}}
$$

where the $P_{\psi_{i}}$ are one-dimensional projection operators on the rays (subspaces of dimension one) generated by the vectors $\psi_{i}$ and $\sum_{i} p_{i}=1\left(p_{i} \geq 0\right)$. Thus, it is clear that $\mathcal{C}$ is a convex set. If the sum in (9) is finite, then $\rho$ is said to be of finite range. Remark that in the infinite-dimensional case the sum in (9) may be infinite in a non-trivial sense. $\mathcal{C}$ is then a set of non-Boolean probability measures, closed by convexity, that is also closed in the norm of $\mathcal{H}$. Let us remind the reader that a lattice is a partially ordered set (also called a poset) in which any two elements have a unique supremum (the elements' least upper bound; called their join) and an infimum (greatest lower bound; called their meet). Lattices can also be characterized as algebraic structures satisfying certain axiomatic identities. Since the two definitions are equivalent, lattice theory draws on both order theory and universal algebra. Semilattices include lattices, which in turn include Heyting and Boolean algebras. These "lattice-like" structures all admit order-theoretic as well as algebraic descriptions. ${ }^{28}$

A very important notion for our purposes is that of $\mathcal{L}_{\mathcal{C}}$, the set of all convex subsets of $\mathcal{C}$. Any element of $\mathcal{L}_{\mathcal{C}}$ will be itself a "probability space," in the sense that it is a set of non-Boolean probability measures closed under convex combinations (not to be confused with the usual mathematical notion of sample space) ${ }^{25}$ It can be shown that $\mathcal{L}_{\mathcal{C}}$ is endowed with a canonical lattice theoretical structure that can be related to quantum entanglement and positive maps. ${ }^{26}$ The meet operation is given by set intersection, the join by convex hull and the partial order by set inclusion. We will use this lattice in order to express the MaxEnt protocol in a different (but equivalent) form. It should be also clear that the lattice operations mentioned above may be trivially defined in any COM and this will be used in Sec. V.

A general (pure) state can be written as

$$
\rho=|\psi\rangle\langle\psi|,
$$


and we denote the set of all pure states by

$$
P(\mathcal{C}):=\left\{\rho \in \mathcal{C} \mid \rho^{2}=\rho\right\} .
$$

This set is in correspondence with the rays of $\mathcal{H}$ via the association

$$
\mathcal{F}: \mathbb{C} \mathbb{P}(\mathcal{H}) \rightarrow \mathcal{C} \quad \mid \quad[|\psi\rangle] \mapsto|\psi\rangle\langle\psi|,
$$

where $\mathbb{C} \mathbb{P}(\mathcal{H})$ is the projective space of $\mathcal{H}$ and $[|\psi\rangle]$ is the class defined by the vector $|\psi\rangle(|\varphi\rangle$ $\sim|\psi\rangle \longleftrightarrow|\varphi\rangle=\lambda|\psi\rangle, \lambda \neq 0$ ). If $M$ represents an observable, its mean value $\langle M\rangle$ is given by

$$
\operatorname{tr}(\rho M)=\langle M\rangle .
$$

Notice that the set of positive operators has the shape of a cone, while the set of trace class operators (of trace one) that of a hiperplane. Thus, $\mathcal{C}$ is the intersection of a cone and a hiperplane, embbeded in $\mathcal{A}$. Such structure (or geometrical convex setting) is susceptible of considerable generalization (see Refs. 6-8 for an excellent overview). In modeling probabilistic operational theories, one associates to any probabilistic system a triplet $(X, \Sigma, p)$, where $\Sigma$ represents the set of states of the system, $X$ is the set of possible measurement outcomes, and $p: X \times \Sigma \mapsto[0,1]$ assigns, to each outcome $x \in X$ and state $s \in \Sigma$, a probability $p(x, s)$ of $x$ to occur if the system is in the state $s$. If we fix $s$ we obtain the mapping $s \mapsto p(\cdots, s)$ from $\Sigma \rightarrow[0,1]^{X}$. We then identify in this way all the states of $\Sigma$ with maps of such a form. Focusing now attention upon their closed convex hull we obtain the set $\Omega$ of possible probabilistic mixtures (represented mathematically by convex combinations) of states in $\Sigma$. One appreciates that we also obtain, for any outcome $x \in X$, an affine evaluation-functional $f_{x}: \Omega \rightarrow[0,1]$, given by $f_{x}(\alpha)=\alpha(x)$ for all $\alpha \in \Omega$. More generally, any affine functional $f: \Omega \rightarrow[0,1]$ may be regarded as representing a measurement outcome and thus use $f(\alpha)$ to represent the probability for that outcome in state $\alpha$.

\section{A. Effects}

In the special case of quantum mechanics, the set of all affine functionals so-defined is called the set of effects. They form an algebra (known as the effect algebra) and represent generalized measurements (unsharp, as opposed to sharp measures defined by projection valued measures). Effect algebras have important applications in the foundations of quantum mechanics ${ }^{29}$ and in fuzzy probability theory. ${ }^{30}$ The specifical form of an effect in quantum mechanics is as follows. A generalized observable or POVM (Refs. 31-33) will be represented by a mapping $E: \mathbf{B}(\mathbb{R}) \rightarrow \mathcal{B}(\mathcal{H})$ such that

1. $E(\mathbb{R})=\mathbf{1}$;

2. $E(B) \geq 0$, for any $B \in \mathbf{B}(\mathbb{R})$;

3. $E\left(\cup_{j}\left(B_{j}\right)\right)=\sum_{j} E\left(B_{j}\right)$, for any disjoint family $B_{j}$.

The first condition means that $E$ is normalized to unity, the second one that $E$ maps any Borel set $\mathrm{B}$ to a positive operator, and the third one that $E$ is $\sigma$-additive with respect to the weak operator topology. In this way, a POVM can be used to define a family of affine functionals on the state space $\mathcal{C}$ (which corresponds to $\Omega$ in the general probabilistic setting) of quantum mechanics, as follows:

$$
\begin{gathered}
E(B): \mathcal{C} \rightarrow[0,1] \\
\rho \mapsto \operatorname{tr}(E \rho) .
\end{gathered}
$$

Effects are then positive operators $E(B)$ which satisfy $0 \leq E \leq \mathbf{1}$ (Refs. 10 and 34). Let us denote by $\mathcal{E}(\mathcal{H})$ to the set of all effects in the quantum theory. We appeal to effects below in order to define a special example of elements of $\mathcal{L}_{\mathcal{C}}$, and also as a generalization of conditions imposed in the MaxEnt protocol. Returning now to the general model of probability states we may consider the convex set $\Omega$ as the basis of a positive cone $V_{+}(\Omega)$ of the linear space $V(\Omega)$. Thus, every affine linear functional can be extended to a linear functional in $V(\Omega)^{*}$ (the dual linear space). It can be shown that there is a unique unity functional such that $u_{\Omega}(\alpha)=1$ for all $\alpha \in \Omega$ (in quantum mechanics, this unit functional is the trace function). Thus, the COM approach speaks of a triplet $\left(A, A^{\sharp}, u_{A}\right)$, where $A$ is 
a space endowed with a strictly positive linear functional $u_{A}$ and $A^{\sharp}$ is a weak-* dense subspace of $A^{*}$, ordered by a chosen regular cone $A_{+}^{\sharp} \subseteq A_{+}^{*}$ containing $u_{A}$. Effects will be functionals $f$ in $A_{+}^{\sharp}$ such that $f \leq u_{A}$.

\section{A GEOMETRICAL EXPRESSION FOR MAXENT}

Our idea is to rediscuss MaxEnt in terms of elements of $\mathcal{L}_{\mathcal{C}}$ associated with conditions on given sets of observables or effects. The main difference between the approach presented here and the original formulation of Jaynes' lies in the fact that we are recasting MaxEnt in the more general geometrical setting of the COM approach, which allows for a purely geometrical reformulation of the principle, extensible to any statistical theory. As we shall see below, our reformulation also allows for establishing an interesting connection between MaxEnt and a lattice theoretical approach ${ }^{26,27}$ and also provides a generalization of the MaxEnt protocol for generalized measurements. In the rest of this work, we restrict ourselves to the finite dimensional case. If $\mathrm{E}$ is an effect and $\lambda$ a real number in the interval $[0,1]$, consider the set

$$
C_{(E, \lambda)}:=\{\rho \in \mathcal{C} \mid \operatorname{tr}(\rho E)=\lambda\} .
$$

Here $C_{(E, \lambda)}$ is a convex set, and so, an element of $\mathcal{L}_{\mathcal{C}} \cdot C_{(E, \lambda)}$, represents all the states for which the probability of having the effect $E$ is equal to $\lambda$. Furthermore, there exists $\mathbb{S}$, a $\mathbb{R}$-subspace of $\mathcal{A}$ (the set of bounded self adjoint operators), such that

$$
C_{(E, \lambda)}=\mathbb{S} \cap \mathcal{C}
$$

and thus, $C_{(E, \lambda)}$ is also an element of $\mathcal{L}$, the lattice induced by the intersection of all closed subspaces of $\mathcal{A}$ and $\mathcal{C} .^{26,27}$ More generally, if

$$
\langle R\rangle=r
$$

then the above equation may be considered as represented by the set of density matrices which serve as a solution of it. The ensuing set is obtained as the intersection of the Kernel of the functional $F_{R}(\rho):=\operatorname{tr}(R \rho)-r \operatorname{tr}(\rho)$ and $\mathcal{C}$. Accordingly, each equation of the form (17) (understood as an equation to be solved) can be represented as an element $C \in \mathcal{L}$, and also as an element of $\mathcal{L}_{\mathcal{C}}$. $C$ is also a closed set, because it is the intersection of the kernel of a functional (which is a closed subspace) and $\mathcal{C} .^{26}$

With such materials at hand, we can now re-express the maximum entropy principle ${ }^{2,3}$ in lattice theoretical form. Our point here is that the set of conditions (1) can be expressed in an explicit lattice theoretical form as follows. Using a similar procedure as in (17), we conclude that each of the equations in (1) can be represented as a convex (and closed) sets $C_{R_{i}}$. In this way, we can now express conditions (1) with the lattice theoretical expression

$$
C_{\text {max-ent }}:=\bigcap_{i} C_{R_{i}}=\bigwedge_{i} C_{R_{i}} .
$$

Now, $C_{\text {max }- \text { ent }}$ is also an element of $\mathcal{L}_{\mathcal{C}}$ (but not necessarily of $\mathcal{L}$ ) and we must maximize entropy on $C_{\text {max - ent }}$. We have thus encountered a MaxEnt-lattice theoretical expression: given a set of conditions represented generally by convex subsets $C_{i}$, one should maximize the entropy in the set $C_{\text {max }- \text { ent }}=\bigwedge_{i} C_{i}$.

\section{GENERALIZATION}

A (generalized) observable in a test space $\Omega$ will be given by a function $F: x \mapsto F_{x}$ from subsets of a given outcome set $E$ into $A(\Omega)$ satisfying

1. $F_{x} \geq \mathbf{0}$;

2. $\sum_{x \in E} F_{x}=u$.

This is the natural extension of the notion of POVM to any COM (extension which includes the more restricted case of projection operator valued measures for the quantum case). 
If $\omega \in \Omega$, we can construct the probability function $p_{\omega, F}(x)=F_{x}(\omega)$, and this pulls back a map $F^{*}: \Omega \rightarrow \Delta(E)$, with $F^{*}(\omega)=p_{\omega, F}$. With this at hand, we can easily generalize probabilities (and so, when they appear, mean values) as linear conditions. With this procedure we will obtain convex sets and proceed similarly as in Sec. IV. For example, suppose that the outcomes of our observable $F$ are represented by the discrete set of real numbers $\left\{f_{i}\right\}_{i \in \mathbb{N}}$. Then we can write the mean value of $F$ in the state $\omega$ as

$$
\langle F\rangle_{\omega}=\sum_{i} f_{i} F_{f_{i}}(\omega)
$$

The probability functions $F_{f_{i}}(\cdot)$ are linear operators defined on $A$ taking values in the interval $[0,1]$ when restricted to states. As they are linear, we can define the functional

$$
\langle F\rangle: A \rightarrow \mathbb{R} \mid\langle F\rangle(a) \mapsto \sum_{i} f_{i} F_{f_{i}}(a) .
$$

If $r$ is a real number, consider the new functional

$$
\langle F\rangle_{r}: A \rightarrow \mathbb{R} \mid\langle F\rangle_{r}(a) \mapsto \sum_{i} f_{i} F_{f_{i}}(a)-r u(a)
$$

then, the Kernel of $\langle F\rangle_{r}$ will be a linear subspace of $A$, call it $\mathbb{S}_{r, F}$. If we now restrict to the elements of $\Omega$ (and any $\omega \in \Omega$ satisfies $u(\omega)=1$ ), we will have that solutions of Eq. (19) can be represented with the convex set

$$
C_{r, F}:=\mathbb{S}_{r, F} \cap \Omega
$$

More generally, even if our measurement outcomes are just labeled by an index set and we are not interested on mean values but only on probabilities (as is generally the case for many POVM's), conditions will have the form

$$
p_{\omega, F}(x)=F_{x}(\omega)=\alpha
$$

and following an analogous reasoning line as above, it can also be put in the form $\mathbb{S}_{\alpha, F} \cap \Omega$, with $\mathbb{S}_{\alpha, F}$ a subspace which depends on the real number $\alpha$ and the observable $F$.

In any case, the construction given above provides the path for generalization of the procedure outlined in Sec. IV to any COM. If $E_{i}$ represent observables and the brackets $\langle\cdot\rangle$ are meant to express either conditions of the form of Eqs. (19) or (23), suppose as given a set of conditions of the form

$$
\left\langle E_{i}\right\rangle=e_{i} \text {; for all } i .
$$

Then, because of the above discussion, the set of conditions (24) may be expressed as a collection of convex subsets of the form $C_{e_{i}}=\mathbb{S}_{e_{i}} \cap \Omega$. And the Jaynes method tells us that we must maximize entropy on the convex set

$$
C_{\text {MaxEnt }}=\bigwedge_{i}\left(\mathbb{S}_{e_{i}} \cap \Omega\right) .
$$

It is important to remark here that our construction does not only restricts to projective measurements, but it includes the possibility of imposing conditions on POVMs. Thus, we provide here a generalization of the MaxEnt protocol for generalized measurements.

We notice that the specific form of the entropy to maximize depends on the specifical COM which is being used. For example, for the particular cases of the quantum theory and a classical model, von Newmann and Shannon entropies are used, respectively, in a natural way. The election of the particular function to maximize on $C_{M a x E n t}$, does not depend on the general formulation but in the specifical characteristics of the model. An interesting question would be to set up general conditions for entropies in any model, but the existence of such conditions is hard to believe, because of the proliferation of entropies and contexts. 


\section{CONCLUSIONS}

In this work, we reviewed the celebrated Jaynes' MaxEnt principle and studied its generalizations of arbitrary probabilistic models via the COM approach. In Sec. IV, we provided new clothing to the maximum entropy principle in the form of lattice theory in terms of convex sets (taking the maximum at set theoretical intersections, i.e., lattice conjunctions). In Sec. V, we used the key fact that a probability function of COM preserves convexity in order to link the COM approach with the results of Sec. IV, this giving MaxEnt a considerable scope-generalization that also includes a protocol for conditions on POVMs in any COM. This kind of generalization may be useful to extend the powerful MaxEnt approach to more general theories, also shedding light onto its mathematical and geometrical structure/background.

\section{ACKNOWLEDGMENTS}

This work was partially supported by the grant PIP Grant No. 1177-CONICET.

${ }^{1}$ L. Brillouin, Science and Information Theory (Academic, New york, 1956).

${ }^{2}$ E. T. Jaynes, Phys. Rev. 106, 620 (1957); 108, 2 (1957).

${ }^{3}$ A. Katz, Principles of Statistical Mechanics: The Information Theory Approach (Freeman, San Francisco, 1967).

${ }^{4}$ A. R. Plastino and A. Plastino, Phys. Lett. A 181, 446 (1993); M. Casas, F. Garcias, A. Plastino, and Ll. Serra, Physica A 213, 376 (1995).

${ }^{5}$ Physics and Probability: Essays in Honor of E. T. Jaynes, edited by W. T. Grandy and P. W. Milonni (Cambridge University Press, New York, 1993).

${ }^{6}$ H. Barnum, J. Barrett, M. Leifer, and A. Wilce, Phys. Rev. Lett. 99, 240501 (2007).

${ }^{7}$ H. Barnum and A. Wilce, Electron. Notes Theor. Comput. Sci. 270, 3 (2011).

${ }^{8}$ H. Barnum, R. Duncan, and A. Wilce, e-print arXiv:1004.2920v1 [quant-ph].

${ }^{9}$ E. Beltrametti, S. Bugajski, and V. Varadarajan, J. Math. Phys. 41, 2500 (2000).

${ }^{10}$ G. Cattaneo and S. Gudder, Found. Phys. 29, 1607-1637 (1999).

${ }^{11}$ S. P. Gudder, Stochastic Methods in Quantum Mechanics (North Holland, New York, 1979).

12 B. Mielnik, Commun. Math. Phys. 9, 55-80 (1968).

${ }^{13}$ B. Mielnik, Commun. Math. Phys. 15, 1-46 (1969).

${ }^{14}$ B. Mielnik, Commun. Math. Phys. 37, 221-256 (1974).

${ }^{15}$ G. Birkhoff and J. von Neumann, Annals Math. 37, 823-843 (1936).

${ }^{16}$ A. N. Kolmogorov, Foundations of the Theory of Probability, translated by N. Morrison (Chelsea, New York, 1950).

${ }^{17}$ M. Rédei, Quantum Logic in Algebraic Approach (Kluwer, Dordrecht, 1998).

${ }^{18}$ J. von Neumann, Mathematical Foundations of Quantum Mechanics (Princeton University, Princeton, NJ, 1955)

${ }^{19}$ P. Goyal, K. H. Knuth, and J. Skilling, Phys. Rev. A 81(2), 022109 (2010).

${ }^{20}$ P. Goyal and K. H. Knuth, Symmetry 3, 171-206 (2011).

${ }^{21}$ R. T. Cox, Am. J. Phys. 14, 1-13 (1946).

${ }^{22}$ R. T. Cox, The Algebra of Probable Inference (The Johns Hopkins, Baltimore, MD, 1961).

${ }^{23}$ D. Buhagiar, E. Chetcuti, and A. Dvurečenskij, Found. Phys. 39, 550-558 (2009).

${ }^{24}$ A. Gleason, J. Math. Mech. 6, 885-893 (1957).

${ }^{25}$ F. Holik and A. Plastino, Phys. Rev. A 84, 062327 (2011).

${ }^{26}$ F. Holik, C. Massri, and N. Ciancaglini, Int. J. Theor. Phys. 51, 1600-1620 (2012).

${ }^{27}$ G. Domenech, F. Holik, and C. Massri, J. Math. Phys. 51, 052108 (2010).

${ }^{28}$ S. N. Burris and H. P. Sankappanavar, A Course in Universal Algebra (Springer-Verlag, Berlin, 1981).

${ }^{29}$ W. Muynck, Contribution to Proceedings of the Workshop, "Beyond the quantum", Leiden, May/June 2006 (pp. 69-79).

${ }^{30}$ S. Gudder and S. Pulmannova, Commun. Math. Univ. Carolinae 39, 645 (1998).

${ }^{31}$ P. Busch, J. Kiukas, and P. Lahti, Math. Slovaca 60(5), 665-680 (2010).

${ }^{32} \mathrm{~T}$. Heinonen, Imprecise measurements in quantum mechanics, PhD-Thesis (Turun Yliopisto, Turku 2005).

${ }^{33} \mathrm{Z}$. Ma and S. Zhu, e-print arXiv:0811.2454v1 [quant-ph].

${ }^{34}$ D. Foulis and S. Gudder, Found. Phys. 31, 1515-1544 (2001). 